\title{
Ethical questions regarding health insurance in India
}

\author{
VINEESH MATHUR
}

Consultant, Orthopaedics, Medanta, The Medicity, Gurgaon 122001 Haryana INDIA e-mail: drvmathur@gmail.com

\begin{abstract}
Improved health and healthcare are of vital concern to the welfare of Indian society. The nascent health insurance system of the country is experiencing an explosive expansion and various models of health insurance provision are under trial by different agencies. Since the country has been relatively late in introducing health insurance, it can study the effects of different systems of healthcare and insurance and develop a system of health coverage which addresses the unique social character of our country as well as the ethical questions of comprehensiveness and inclusion. This article seeks to explore these issues in detail.
\end{abstract}

Disease is inevitable in the life of every individual, and brings along with it the burden of physical pain, emotional distress, and financial strain. Cutting-edge technology has brought tremendous improvements in the quality and of life and lifespan but at the cost of affordable healthcare for the majority.

Surveys have shown that ill health and its related costs are the biggest causes of indebtedness in rural India, with one fourth of the rural population in the poorest groups falling into a debt trap to pay for healthcare.

In an ideal world, the state and the community should shoulder the burden of caring for sick individuals till they return to productive health and work. The government of India has proved to be a poor provider of health services, thus creating a gap which private health services have exploited. This has necessitated the development of mechanisms to provide an adequate financial buffer to cover the costs of treatment for a large part of the population.

The "return on capital employed" is considered in both social and financial terms, and thus deciding the model of payment creates contradictions that give rise to questions regarding the nature of insurance in India.

\section{Current state of health insurance in India}

In the current situation healthcare is financed through general tax revenue, community financing, out-of-pocket payment and social and private health insurance schemes.

The per capita total expenditure on health in India is US\$ 36, of which the per capita government expenditure on health is US\$ 5 (1). India spends about $4.9 \%$ of its GDP on health (2). The breakdown is public expenditure $(0.9 \%)$; private expenditure (4.0\%). The private expenditure can be further classified as out-of-pocket (OOP) expenditure (3.6\%) and employees/ community financing (0.4\%). (3)

Over the last 50 years we have developed a large government health infrastructure with more than 150 medical colleges, 450 district hospitals, 3,000 community health centres, 20,000 primary health centres and 130,000 sub centres. In addition there are a large number of health facilities run by private, and non governmental organizations (NGOs), and private practitioners scattered throughout the country.

India is a low-income country with $26 \%$ of the population living below the poverty line, and $35 \%$ of the population illiterate and with skewed health risks. Yet the private sector is the dominant sector; $50 \%$ of those seeking indoor care, and $60-70 \%$ of those seeking ambulatory care, do so from private health facilities which include paramedical providers and even quacks. Despite the higher cost, a majority of both rural and urban Indians prefer private care because of the perceived inferior quality of public care. The entire burden of cashless, prepaid private care is shared among $\mathbf{5 0 0}$ odd private care providers in the country who are approved by insurance companies for this purpose.

\section{Viability, coverage and types of insurance available}

The existing schemes can be categorised as:

1. Voluntary health insurance schemes or private-for-profit schemes;

2. Employer-based schemes

3. Insurance offered by NGOs / community based health insurance, and

4. Mandatory government-run health insurance schemes.

With less than $11 \%$ of the country's population covered by any formal health insurance, and only $1 \%$ by private medical insurance, the product has been seen to be made for the privileged, and as a luxury(4).

Currently, India chooses to have a healthcare payment system which is somewhere between the American and German systems. The American system of healthcare is a private, for profit system where the owners of insurance companies and the providers are largely privately owned. Premiums are collected from the insured, their employers and, for certain diseases, federal funds. The amount of premium is based on actuarial risk, and selections and refusals do occur; reimbursement 
to providers is based on costs and a per procedure basis. The nature of subsidy is from the healthy to the sick; choice of procedures and providers is governed by health management organisations. Co-payments are high, and there is competition between different insurers. Regulation of the schemes is largely governed by good business practice with minimal interference from the government. This system is currently groaning under the weight of progressively high costs and is being subjected to introspection and reform. About $70 \%$ of the population is covered by one or the other scheme (1).

In contrast to this, the German system is funded by sickness funds collected by workers; coverage is very high - more than $99 \%$ of the population; and contribution is made as a percentage of one's income. No selections or refusals are allowed and reimbursement is on a per-capita, per case basis. The nature of subsidy is socialistic - from the healthy to the sick, from the high income to the low income, from the young to the old and from small families to big families. The choice of providers is limited, coverage is good and co-payment is low. Generally accepted good practices and treatments are followed and there is self regulation of the companies. Shortfalls are paid out of federal funds. Inflation in costs is limited. The system, however, assumes the presence of excellent governmentaided or -owned hospitals, a robust and efficient payment system between the government and hospitals, and a degree of economic strength, and high socioeconomic indices which reduce the overall claims. Such a system also exists in Canada and to some extent in the United Kingdom (6).

In 2004 the Indian government introduced the Universal Health Insurance (UHI) scheme, which was aimed at those living below the poverty line. The UHI, also referred to as the "Government Rupee-a-Day" scheme (because the annual premium is Rs 365 per person), was largely unsuccessful at attracting the poor, mainly because the insurance companies, that are required to implement the scheme, found it "loss-making" because of adverse selection, and did not market or sell it sufficiently. There is no mechanism or infrastructure for collecting mandatory premia among the large informal sector. There is insufficient and inadequate information about the scheme.

The recently relaunched Rashtriya Swasthya Bachat Yojna is more successful as it has enrolled 1.95 crore beneficiaries as of 2010. The budget is largely state-funded with the central and state governments contributing $75 \%$ and $25 \%$ of the premium respectively, and a small enrollment fee of Rs 30 being charged from the beneficiaries. The scheme is more in the nature of a subsidy with little participation from the beneficiaries; it is targeted at people living below the poverty line and hence its financial success is not ascertainable. Factors that run against the scheme are its relatively low coverage of Rs 30,000 and its continued focus on private hospitals (7).

Self-governed "micro insurance schemes" can create tailormade policies for people with low incomes. These differ from commercial insurance policies in that the policy is a low-valued product with modest benefits, modest premium amounts, and simpler documentation. Advantages of member-owned self-insured schemes include lower risk of moral hazard since the membership has an economic stake in the risk pool and "one for all and all for one" solidarity, conducive to improved participation. Local knowledge and administration of selfinsured schemes results in better service such as reduced time required to process claims. Lastly, all profits or surpluses remain with the members and can be used to build up additional reserves or to increase benefits in the future.

The International Labour Organization estimated in 2005 that there were 51 micro-insurance schemes operating in India covering approximately 5.1 million people (5). The majority of them operate within a partner-agent model in which an insurance company is the "partner" insuring the risk of the group, and with a second organisation such as a community based organisation (CBO) acting as implementer or "agent" handling marketing and administration. However, some are entirely self-insured and some are a combination of the first two, partnering with a health insurance company which assumes part but not all of the risk with the CBO. The performance of micro insurance schemes varies widely and cannot be considered exemplary. Efficiency as measured by administration costs, including marketing, is not properly documented. There is evidence from the Karnataka scheme that increased size may reduce these costs as a percentage of premia. Schemes with comprehensive coverage are heavily subsidised while for-profit microfinance insurance channels such as BASIX provide very inadequate coverage. Most of the schemes do not emphasise health education, prevention or primary care.

There is also a need to evolve criteria to be used for deciding upon target groups, who would avail of social health insurance scheme/s, and also to address issues relating to whether indirect costs would be included in health insurance.

\section{Underwriting and regulatory issues}

The health benefits environment in India went through a substantial change in the early 1980s, when four governmentowned general insurance companies in India launched the Group Mediclaim Policy - a group hospitalisation and surgical reimbursement policy - for the private sector corporate market. The healthcare delivery market shifted with strong leadership from the private sector, especially in the urban centres. Group insurance helped employees access the newly emerging private healthcare services.

Unfortunately, at least initially the group Mediclaim coverage was sold more as an "accommodating business" to corporates for acquiring the highly profitable property and casualty businesses (because of government-mandated premium rates). The product was intentionally sold at a loss, and by the late 1990s claims were almost double the amount of premia. This loss was accepted by insurance companies since the loss made was small compared to the profits from the other business brought in. 
Health insurance products are generally complicated and it has been suggested that general insurers who deal in the non-life insurance market, which is dominated by mandated insurance such as accident, fire and marine, do not have expertise in pricing and administering health insurance, which is why this scheme is not popular. For example, Mediclaim represents just $10 \%$ of the overall business of General Insurance Corporation and its subsidiaries; hence they have also not focused their attention in this area. Even the latest yearly financial reports of these companies lump health insurance with the "miscellaneous" group which is the biggest loss-making group of the company. It should also be recognised that because of technicalities of the health service business there are a number of cumbersome rules which have hampered the acceptance of the scheme.

It is reported that in a number of cases applicants of older ages have been refused admission to the Mediclaim scheme due to the unnecessary conservatism of companies since it is felt that the outgo of these schemes will be more than the premium collected.

Companies are also refusing to upgrade or increase the sum assured for older patients.

One reason why pricing anomalies prevail is that insurance companies lack the data they need to assess health risks accurately. In addition, today's insurance products work on an indemnity basis i.e. they look at health only as a series of payable events. It covers hospitalisation costs, which could be catastrophic as it gives precedence to tertiary level healthcare and encourages inpatient treatment. This is coupled with the fact that in the absence of any costing mechanisms, there is difficulty in calculating the premium, and the easiest and most illogical way wins viz, the reverse auction system or the lower bid.

Suggested ways to cover the gaps in regulatory issues with private insurers are as follows.

- Lay down a minimum regulatory definition of a pre-existing illness or condition to provide clarity and uniformity of its interpretation, including prescribing maximum "look-back" and "look-forward" periods.

- Ensure that a health insurance contract is incontestable after it has remained in force for a specified period of time from the date of inception. This would ensure that the "preexisting disease" clause would cease to be operative after a certain period of time.

- Prohibit post-claims underwriting in order to allow automatic renewal of policies even after claims have been made. Appropriate front loading of premia in the initial years could be allowed with a gradual reduction in premia after a certain holding and no claim period.

- Require insurers to offer both group and individual policies in order to remove the present bias towards corporate and group insurance and also allow insured people to carry their insurance with them even if they changes jobs or occupations.
- Prescribe standards on point-of-sale and after-sales disclosures specific to health insurance.

- Adopt reserving rules specific to the different types of health insurance contracts.

- Lay down additional regulations particularly for medical expense covers that prescribe the following:

- availability or accessibility;

- transferability or portability, and

- continuity (renewability and cancellation)

- Establish rules on over-insurance in the case of individual covers, and coordination of benefits, in the case of group covers.

- Refine the micro insurance regulations in the following aspects:

- eliminate the minimum and increase the maximum sum insured:

- eliminate or relax the "one-partner-one-agent rule";

- eliminate commission caps and

- expand the authority of micro insurance agents who are also organisers of health micro insurance to specifically include, among others, enrolment of members, collection of premia and post-sales servicing including settlement of claims.

- Adopt special agent licensing rules to allow officers and staff of panchayats, rural health practitioners and postmen to solicit health micro insurance.

Since the social health model seems better suited to a country like India, other mechanisms that could be used to encourage social insurance are:

- encouraging the evolution of specialised medical insurance corporations which includes separating and then hiving off the health business of companies into separate companies;

- using part of the central health outlay in capitalising these companies;

- encouraging companies to collaborate and develop regional areas of competence;

- encouraging mergers of smaller health companies with larger ones to ensure financial stability;

- encouraging corporates to use these stand-alone health corporations by offering tax benefits and regulatory muscle; and

- using a national task force to rapidly increase the percentage covered to about $20 \%$ to generate a positive feedback cycle for growth.

The Insurance Regulatory and Development Authority will need to evolve mechanisms by which it puts some kind of statute in place to ensure that private insurance companies do not skim the market by focusing on rich and upper-class clients, in the process neglecting a major section of India's population.

The regulators should also encourage NGOs, co-operatives and other collectives to develop products for the poor as well as for the middle class employed in the services sector, such as 
education, transportation and retailing, and the self employed. This could be run on a no-profit-no loss basis.

While following the basic principles of insurance is necessary for success, health insurance is a special branch of insurance. It includes concepts such as prepayment for health services, through which large portions of the insured are not only expected but are also encouraged to use primary care services so as to reduce the risk for future, often costlier, services. Prenatal care and well-baby care are excellent examples of services that, when properly provided, can actually save the insurer from future claims.

\section{Administrative and servicing issues}

Insurance claims are frequently rejected due to minor, technical reasons leading to disputes. Conditions and various points included in insurance policy contracts are generally not negotiable and are binding on consumers. There is no analysis on what is and is not fair practice. Given that insurance companies are large monopolies, the consumer is treated as secondary and does not have an opportunity to negotiate the terms and conditions of a contract. Insurance companies may not strictly follow the conditions in all cases and this creates confusion and disputes. This has been poignantly brought out in the recent impasse where individual policy holders were denied insurance on a cashless basis, though their policies entitled them to cashless insurance.

There are several exclusions, for treatment of sexually transmitted diseases, AIDS, delivery and maternal conditions, etc. These are not socially and ethically acceptable. Insurance companies must take care of all the risks related to health though they may charge an additional premium for certain conditions.

The recent impact of controlling costs does not focus on improving the quality of care. Mediclaim has been modified in ways that make it less a programme to control the cost of care and more a reimbursement target for providers. As a result, policy holders pay higher charges for services than those without insurance. Products such as "dreaded disease insurance" and "critical illness" policies may make money for the insurance companies but usually much of the focus of the existing schemes is on hospital expenses.

Private healthcare providers continue to operate in an almost unhindered manner. The growth of health insurance increases the need for licensing and regulating private healthcare providers and developing specific criteria to decide upon appropriate services and fees.

No one in the industry is taking responsibility to develop knowledge and awareness of health insurance among the public; nor is specific expertise in health insurance being developed within the private sector, an expertise that is essential to dealing effectively with providers of healthcare services.
Indian companies writing health insurance seem to have focused on controlling claims payout by following strategies designed to minimise the insured person's ability to collect on claims. There is an excessive emphasis on disqualification because of pre-existing conditions and post-claim underwriting. Because of these practices health insurance has become one of the largest litigation areas for insurers, exceeded only by motor third party cases.

Private insurers' administrative costs may be as high as $40 \%$ of total premiums, double the benchmark target of $20 \%$. Much of the actual administrative work is being done by third party administrators (TPAs) but at a fixed rate that varies regionally from 4 to $5 \%$ of premia. Further, TPAs have little incentive to control costs, and big incentives to collude with providers.

\section{Provider-related issues}

A mechanism to contain provider behaviour and costs can only be implemented by developing mechanisms in which government and individuals can together pool their funds.

Health insurance is also different in that the providers of care whose services are covered by insurance enjoy high prestige, income and political influence. Also, because of their unique skills, the state often grants these providers extensive independence and autonomous power over medical decisions. Their efforts are focused on preserving one of the most precious of human assets, life itself, and it requires special skills to work with them to make sure insurance enhances access and the quality of care while keeping costs reasonable.

There are reports of fraud and manipulation by clients and providers, which have implications for the growth and development of this sector. Monitoring systems are weak and there are chances that if the doctor and patient collude, they can do more harm to the system. Various mechanisms like identity issues, masking, bundling, unbundling, upgrading, etc are used by providers to charge more from insurers and thus defeat the purpose of providing access to good quality healthcare with adequate facilities and skilled personnel at an affordable cost.

Currently the schemes of hospitalisation and payment also seem to infringe on the individual's right to choose a service provider.

It is also a moot legal point whether insurers can decide how much to pay for a particular disorder or whether insurance should restrict its role to financial coverage or act as a treatment modifier and healthcare guide.

\section{Confidentiality issues}

Medical information is sensitive information since it affects the life, employability, career, earnings, marital prospects, and social standing of a person. As the pool of insurable prospects increases, insurers will sit on a mountain of personal data which is potentially damaging to the individual. Correspondingly, a system of data banks should be created where the information 
regarding individuals should be relatively inaccessible except for purely commercial reasons in pricing and administering insurance products.

\section{Dangers}

The main danger in the health insurance business is that private companies will cover the risk of the middle class who can afford to pay high premiums. Unregulated reimbursement of medical costs by insurance companies will push up the prices of private care and a large section of India's population who are not insured will be at a relative disadvantage as they will, in future, have to pay much more for private care

Unless privatisation and development of health insurance is managed well it will have a negative impact on healthcare especially for a large segment of the population in the country.

Health insurance is different from other segments of the insurance business and is more complex because of serious conflicts arising out of moral and ethical considerations to treat. For example, experiences from other countries suggest that the entry of private firms into the health insurance sector if not properly regulated, has adverse consequences for the costs of care, equity, consumer satisfaction, fraud prevention, and ethical standards. Analysis of Mediclaim data, though fragmented, indicates a wide variation of charges for the same operation in the same city. Anecdotal evidence from doctors also indicates that charges are increased if patients are insured. All these effects will tend to increase the prices of private healthcare, thus hurting the uninsured.

\section{Positive factors}

The demand for health insurance has been growing at a rate of $25 \%$ per year, driven by rapidly increasing awareness, and is going to develop even more rapidly in the future. The challenge is to see that this benefits the poor and the weak in terms of better coverage and healthcare services at lower costs without the negative aspects of cost increase and overuse of procedures and technology in the provision of healthcare. The experience from other places suggests that if health insurance is left to the private market it will only cover those who have a substantial ability to pay, leaving out the poor and making them more vulnerable. For India this represents an opportunity to proactively make efforts to develop social health insurance in a virgin environment; it can ensure universal coverage and equal access to all and implement cost-controlling measures such as prospective per capita payment to providers.
The current effort by the government to develop the Universal Identification Number will also aid the development of a sound database for analysis and research.

\section{Conclusion}

There is an urgent need to document global and Indian experiences in health insurance so that different financing options are developed for different target groups. The wide differentials in demographic and epidemiological status and in the delivery capacity of health systems are a serious challenge to a nationally mandated health insurance system. but one that has to be tackled head on. Health policymakers and health systems research institutions, in collaboration with economic policy study institutes, need to gather information about the prevailing disease burden at various geographical regions; develop standard treatment guidelines; undertake costing of health services for evolving benefit packages to determine the premium to be levied and subsidies to be given; and map the healthcare facilities available and the institutional mechanisms which need to be in place for implementing health insurance schemes. Skill building for the personnel involved, and capacity-building of all the stakeholders involved, will be critical components to ensure the success of any health insurance programme. The success of any social insurance scheme will depend on its design and the implementation and monitoring mechanisms which will be set in place. It will also call for restructuring and reforming the health system.

\section{References}

1. Bhat R, Mavlankar D. Health insurance in India: opportunities, challenges and concerns. Ahmedabad: Indian Institute of Management: 2000.

2. World Health Organization. World Health Report 2007. Geneva: WHO; 2007.

3. Ellis RP, Alam M, Gupta I. Health insurance in India: prognosis and prospects. Econ Pol Wkly. 2000 Jan 22; 35(4): 207-16.

4. Government of India. Annual Report, 2006-7. New Delhi: Ministry of Health \& Family Welfare; 2007.

5. Roth J,Churchill C, Ramme G,Namerta.Microinsurance and microfinance institutions: evidence from India [Internet].Place unknown:International Labour Organization. 2005 Sep [cited 2010 Dec 13]. 48p. Available from http://www.ilo.org/employment/Whatwedo/Publications/lang--en/ docName--WCMS_12347/index.htm

6. Mattison N. Putting policies in context - achieving and sustaining better health care quality. Eurohealth. 2003; 9(3):7-12.

7. Government of India. Rashtriya Swasthya Bima Yojna [Internet]. New Delhi:Government of India: [date unknown, cited 2010 Dec 13]. Available from: www.rsby.gov.in

\title{
PLEASE NOTE THE CHANGE IN IJME'S E-MAIL ADDRESSES
}

\author{
Editorial correspondence ijme.editorial @gmail.com
}

Subscription correspondence ijmemumbai@gmail.com 